\title{
Virtual septal myectomy for preoperative planning in hypertrophic cardiomyopathy
}

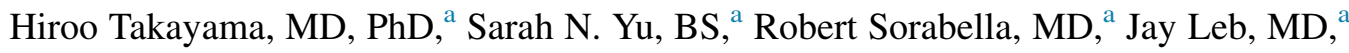

Todd C. Pulerwitz, MD, ${ }^{a}$ Cathleen Cooper, MS, ${ }^{a}$ Melissa Argenio, NP, ${ }^{a}$ Yuichi J. Shimada, MD,

Shepard Weiner, MD, and Jonathan N. Ginns, MD $^{\mathrm{b}}$

\section{ABSTRACT}

Objective: Although septal myectomy (SM) is the preferred treatment for medication-refractory obstructive hypertrophic cardiomyopathy, the procedure remains subjective. We have developed a virtual myectomy (VM) technique using 3-dimensional reconstruction of gated cardiac computed tomography (CT) to assist intraoperative objective assessment of the adequacy of the resection.

Methods: We retrospectively reviewed patients 15 patients who underwent a SM guided by preoperative VM at our program between March 2016 and July 2017. Gated cardiac CT was performed to allow delineation of the left ventricular (LV) myocardium at end-diastole to replicate the cardioplegic myocardial arrest (90\%$95 \%$ RR interval). SM was performed to attain resection volume predicted by VM. Retrospective, blinded VM also was performed with fixed parameters to determine relationship between ideal (VM1) and conservative (VM2) VM and actual resection.

Results: Mean patient age was $52.1 \pm 10.6$ years, $27 \%$ were male, and $80 \%$ had New York Heart Association class 3 or 4. Preoperative mean peak LV outflow tract gradient was $79 \mathrm{~mm} \mathrm{Hg}$ (range 47-82). In-hospital mortality was $0 \%$. Mean postoperative LV outflow tract gradient was $13 \mathrm{~mm} \mathrm{Hg}$ (11-19). Gated cardiac CT was performed with mean phase $94 \%$ ( $86 \%-98 \%)$. Mean total LV myocardial volume was $226 \mathrm{~cm}^{3}$ (146-365) and volume of the asymmetric portion of the LV was $19 \mathrm{~cm}^{3}$ (5.2-48.8). Actual surgical resection volume was $6.2 \pm 1.7 \mathrm{~cm}^{3}$. Retrospective VM1 and VM2 performed postoperatively blinded to surgical results were $12.8 \mathrm{~cm}^{3}$ (4.8-29.23) and $6.7 \mathrm{~cm}^{3}$ (3.5-13.2), showing a modest correlation $\left(\mathrm{R}_{1}=0.44, \mathrm{R}_{2}=0.56\right)$ with actual myectomy.

Conclusions: Three-dimensional CT and VM can be a viable addition to preoperative assessment of patients with obstructive hypertrophic cardiomyopathy. (J Thorac Cardiovasc Surg 2019;158:455-63)

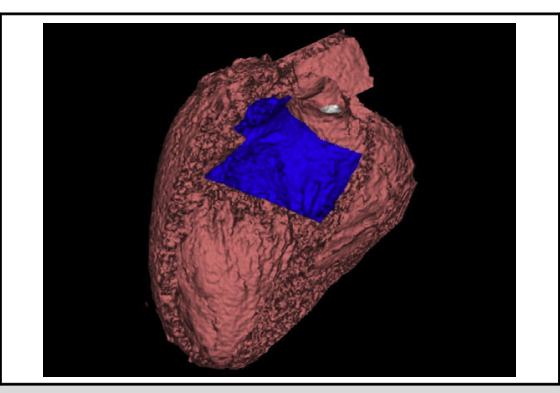

Lateral view of left ventricle $3 \mathrm{D}$ reconstruction with virtual myectomy resection (blue).

\section{Central Message}

$3 \mathrm{D}$ virtual myectomy is a novel technique with the potential for use as an objective preoperative assessment tool for septal myectomy.

\section{Perspective}

Although septal myectomy is the preferred treatment for patients with obstructive hypertrophic cardiomyopathy refractory to medical therapy, it is a challenging procedure that relies on a surgeon's subjective assessment through a limited transaortic surgical view. We have developed a 3D virtual myectomy technique to assist in preoperative planning and intraoperative assessment for septal myectomy.

See Commentary on page 464.
Hypertrophic cardiomyopathy (HCM) is a prevalent disease affecting up to 1 in 200 people. ${ }^{1}$ In total, $60 \%$ to $80 \%$ of patients with HCM develop some degree of left ventricular outflow tract (LVOT) obstruction during the disease process, and septal myectomy (SM) is the preferred therapy

\footnotetext{
From the ${ }^{a}$ Columbia University Medical Center, New York Presbyterian Hospital, New York, NY; and ${ }^{b}$ Weill Cornell Medical Center, New York Presbyterian Hospital, New York, NY.

Received for publication June 15, 2018; revisions received Oct 10, 2018; accepted for publication Oct 12, 2018; available ahead of print Dec 18, 2018.

Address for reprints: Hiroo Takayama, MD, PhD, Division of Cardiothoracic and Vascular Surgery, Columbia University Medical Center, 177 Fort Washington Ave, New York, NY 10019 (E-mail: ht2225@cumc.columbia.edu). $0022-5223 / \$ 36.00$

Copyright (c) 2018 by The American Association for Thoracic Surgery https://doi.org/10.1016/j.jtcvs.2018.10.138
}

for those who remain severely symptomatic despite maximal tolerated medical therapy., ${ }^{2,3}$ Achieving an adequate length and width of resection of the hypertrophic interventricular septum is technically demanding and requires significant surgical expertise and experience. The impact of operator surgical volume in achieving safe, effective myectomy is emphasized in the 2011 American College of Cardiology/American Heart

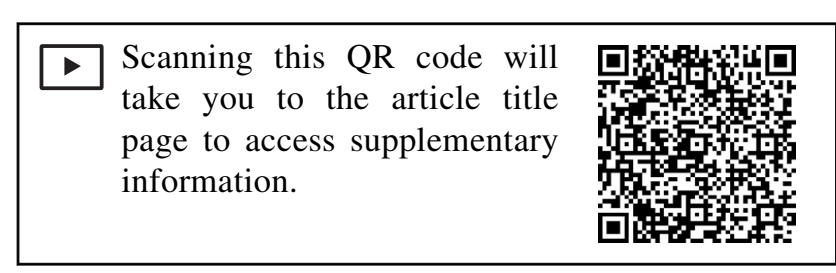




$$
\begin{aligned}
& \text { Abbreviations and Acronyms } \\
& \begin{aligned}
\text { 3D } & =3 \text {-Dimensional } \\
\mathrm{CPB} & =\text { cardiopulmonary bypass } \\
\mathrm{CT} & =\text { computed tomography } \\
\mathrm{HCM} & =\text { hypertrophic cardiomyopathy } \\
\mathrm{LV} & =\text { left ventricular } \\
\mathrm{LVOT} & =\text { left ventricular outflow tract } \\
\mathrm{MR} & =\text { mitral regurgitation } \\
\mathrm{MRI} & =\text { magnetic resonance imaging } \\
\mathrm{SM} & =\text { septal myectomy } \\
\mathrm{TEE} & =\text { transesophageal echocardiogram } \\
\mathrm{TTE} & =\text { transthoracic echocardiogram } \\
\mathrm{VM} & =\text { virtual myectomy }
\end{aligned}
\end{aligned}
$$

Association HCM Guidelines. ${ }^{4}$ Currently, assessment of the adequacy of myocardial resection relies on the surgeon's subjective assessment through a limited transaortic surgical field. Even in experienced hands, up to $20 \%$ of patients may require repeat aortic crossclamping for additional resection. ${ }^{5}$ Recurrence or residual LVOT gradient due to insufficient depth or width of resection at the initial SM may necessitate repeat intervention. ${ }^{2,6}$ The limited number of surgical experts located in a few geographically dispersed high-volume centers may discourage some patients from seeking specialized care and/or referring physicians from pursuing this valuable therapy.

Although the surgical literature is replete with descriptions of optimal technique to perform SM, the approach remains somewhat controversial, with many variations described on how to achieve the best surgical outcome. ${ }^{7-9}$ Therefore, an objective tool to assess preoperative left ventricular (LV) anatomy in HCM is necessary to guide surgical intervention. The mainstay of preoperative imaging assessment remains transthoracic echocardiography (TTE), although this modality can significantly mislead in preoperative assessment with regard to the location and severity of LV thickness. ${ }^{10}$ Alternative imaging approaches include intraoperative transesophageal echocardiography (TEE) and cardiac magnetic resonance imaging (MRI). ${ }^{11,12}$ However, TEE is invasive and can only be performed with sedation or anesthesia, and MRI has limitations in patients with pacemakers, claustrophobia, or irregular rhythms such as atrial fibrillation. This raises the question of whether additional imaging modalities are needed that can guide surgical decision-making both preoperatively and intraoperatively. In this context, we have begun using 3-dimensional (3D) computed tomography (CT) to perform virtual myectomy (VM) to assist in surgery. The objectives of the present study are to describe the use of cardiac CT with VM as a preoperative assessment tool and describe outcomes of SM with this technique.

\section{METHODS \\ Patient Selection}

This study is a retrospective review of 15 patients who underwent SM with preoperative cardiac CT and VM in our HCM program between March 2016 and July 2017. Preoperative demographics, surgical procedural details, postoperative outcomes, and detailed information on cardiac CT and echocardiogram were obtained. The institutional review board of Columbia University Medical Center approved the study. Consent was waived due to the retrospective nature of the study.

\section{Patient Assessment, Management, and Indication for Surgical SM}

Upon referral to our program, initial investigations included electrocardiogram and TTE. Resting gradient across the LVOT was assessed with and without Valsalva maneuver. Stress echo was performed to assess exercise capacity or degree of obstruction with activity where indicated. Cardiac MRI was performed in selected cases primarily to evaluate for gadolinium enhancement of the myocardium to guide implantable cardioverter defibrillator placement or where inadequate TTE images were obtained. Patients with symptomatic LVOT obstruction were managed with intensification of pharmacological therapy with a beta-blocker and/or calcium channel blocker. For patients who remained symptomatic despite maximized beta-blocker and calcium channel blocker use, disopyramide was used when appropriate. Septal reduction therapy was offered to patients who remained symptomatic despite maximal tolerated medical therapy. Recommendation for SM or alcohol septal ablation was made based on discussion by our multidisciplinary HCM program. In general, surgical therapy was recommended for lower risk surgical candidates. Preoperative assessment included coronary angiography with LVOT pressure measurement at rest and with premature ventricular contraction provocation.

\section{Preoperative Planning With Cardiac CT and VM}

In January 2016, gated computed tomography angiography of the heart was introduced as part of the preoperative workup. Using a 320-detector row volumetric scanner (Aquilion One; Toshiba America Medical Systems, Otawara, Japan), a cardiac scan was performed with a prospective, 350-millisecond duration electrocardiogram-gated volume scan of the heart centered at the $90 \%$ of the RR interval (late diastole). A triphasic, intravenous injection of $80 \mathrm{~mL}$ of iohexol $350 \mathrm{mg}$ iodine/mL (Omnipaque; GE Healthcare, Princeton, NJ) was administered at a rate of $4.0 \mathrm{~mL} / \mathrm{s}$ for 15 seconds followed by $20 \mathrm{~mL}$ of iodinated contrast at a rate of $2.0 \mathrm{~mL} / \mathrm{s}$ and flushed with $30 \mathrm{~mL}$ of saline flush at $2.0 \mathrm{~mL} / \mathrm{s}$. During an inspiratory breath-hold, single-volume acquisition was performed with prospective electrocardiographic triggering and 240 to $320 \times 0.5 \mathrm{~mm}$ collimation. Tube current and potential were selected by either the supervising physician or automated software with the goal of maximizing image quality and reducing radiation exposure. Real-time bolus tracking in the descending thoracic aorta was used for scan timing. Breath-hold and scan were initiated at 130 and 180 Hounsfield units, respectively, with manual triggering used if necessary.

The obtained images were postprocessed with Vitrea software (Vital Images, Minnetonka, Minn) to identify areas of LV hypertrophy and surrounding structures including the coronary arteries, aortic valve cusps, left trigone, mitral valve leaflets, muscular septum, membranous septum, and the anterolateral and posteromedial papillary muscles. The specific benefits of this software package are that it allows rapid segmentation of the end diastolic image, visualization of anatomical features, as well as facilitating performance of VM, measurement of wall thickness and 

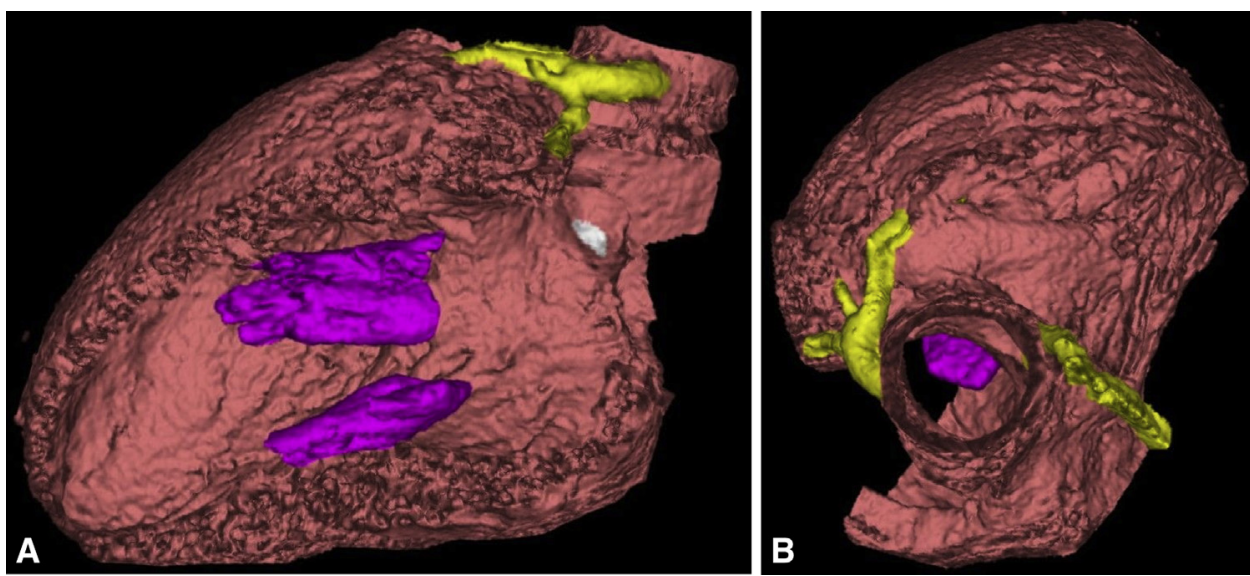

FIGURE 1. 3-Dimensional reconstruction of the left ventricle. A, Lateral view (lateral wall removed to visualize septum, apex on the left of image) and B, surgical transaortic view (oriented with left coronary artery to the left of the image). Left ventricular myocardium is shown in soft pink; papillary muscles in bright pink; membranous septum in white; and coronary arteries in yellow.

distances to important landmarks such at the aortic valve and the membranous septum/conduction system. In addition, depth of resection to avoid VSD or free wall rupture is easily performed and relayed to the surgeon. Total volume of resection is easily calculated to provide a target resection for the surgeon.

$3 \mathrm{D}$ reconstruction was performed to visualize the relevant surgical anatomy (Figure 1, $A$ and $B$, Video 1). Total LV myocardial volume was calculated, as was total volume of asymmetric hypertrophied myocardium (Figure 2). In addition, multiplanar views of the left ventricle were used to measure segmental septal thickness and to perform VM (Figures 3, A and $B$, and 4). Preoperative VM was performed during a multidisciplinary meeting between cardiologists and surgeon of our HCM team, and joint conclusion about areas of hypertrophy requiring resection as well as depth of resection and resection volume were discussed and planned.

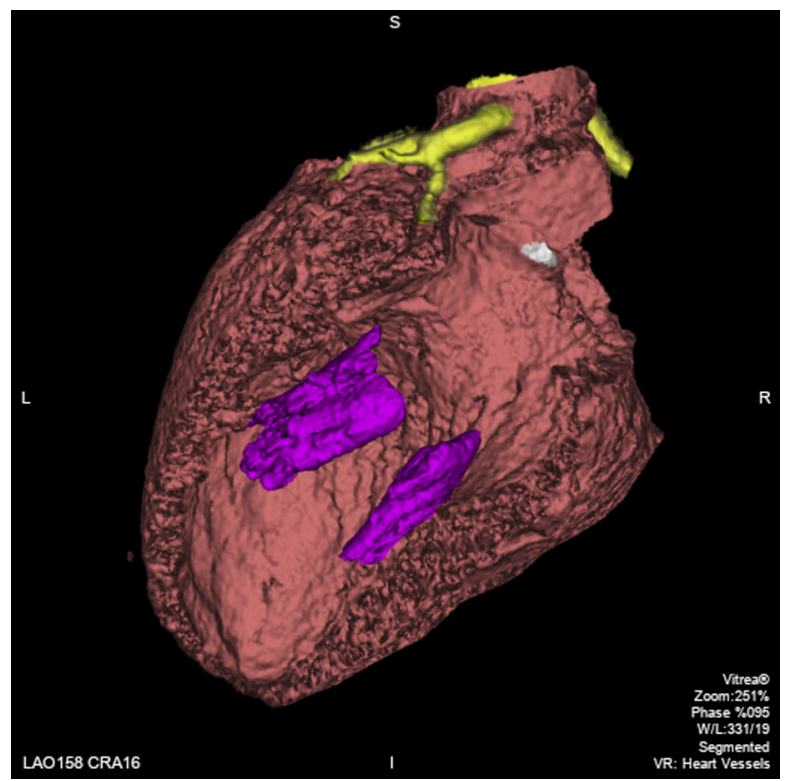

VIDEO 1. Anatomical relationship between papillary muscles, the septal band, and the coronary arteries and membranous septum. Video available at: https://www.jtcvs.org/article/S0022-5223(18)32934-9/fulltext.

\section{Performance of Blinded Retrospective VM}

To compare SM with an idealized VM, blinded VM was retrospectively repeated by 1 cardiologist (J.G.) without input by the surgeon. This was conducted in 2 standardized fashions, VM1 (idealized resection) and VM2 (conservative resection). VM1 resected all asymmetric LV myocardium within typical surgical boundaries, beginning at the left trigone, extending at its basal limit rightward no further than the midpoint of the

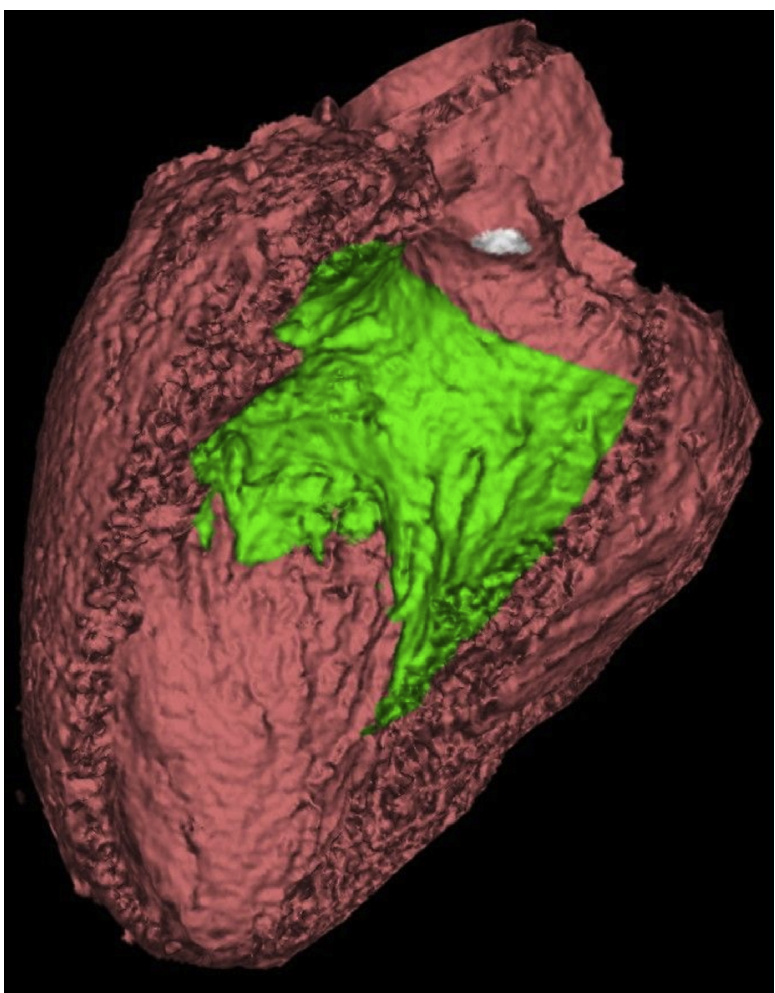

FIGURE 2. 3-Dimensional reconstruction of the left ventricle from a lateral view (lateral wall removed). Total asymmetrically hypertrophied myocardium is shown in green ("septal band"); left ventricular myocardium in soft pink; and membranous septum in white. 


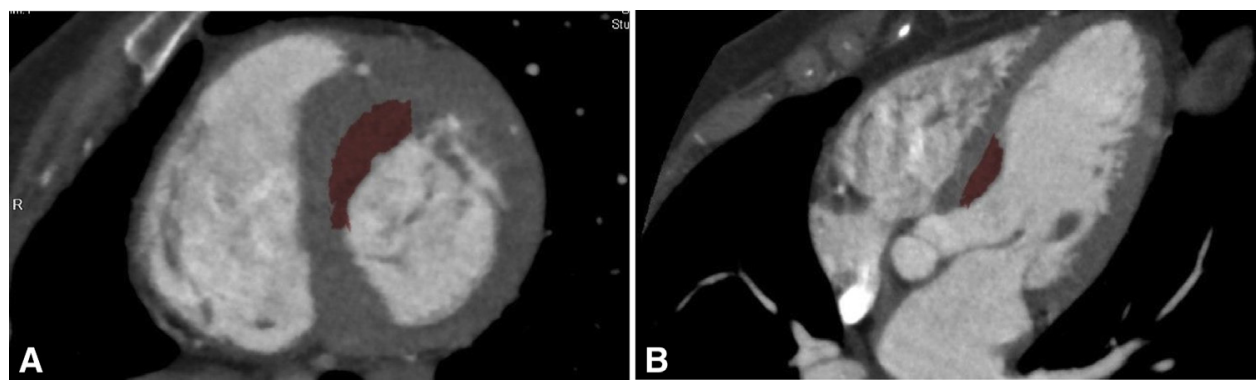

FIGURE 3. Area of planned myectomy (tinted red) is visualized on the (A) short axis and (B) long-axis views of left ventricle/right ventricle on gated computed tomography.

base of the right coronary cusp, proximally no closer than $1 \mathrm{~cm}$ from the aortic annulus, and extending apically no further than $1.5 \mathrm{~cm}$ beyond the posterior papillary muscle head (Figure $5, A$ ). VM2 was similar to VM1; however, depth of resection was limited $1 \mathrm{~cm}$ of the wall thickness excised or $1 \mathrm{~cm}$ of wall thickness remaining, whichever resection thickness was thinner, to simulate the surgeon avoiding creation of ventricular septal defect or LV free wall perforation. In addition, areas deemed surgically inaccessible due to being obscured behind papillary muscle bodies or trabeculations were not considered resectable. VM2 resection was also limited apically and inferiorly by the proximal tip of the posteromedial papillary muscle head (Figure 5, B, Video 2).

\section{Surgical Technique}

SM was performed to resect myocardial volume predicted by preoperative multidisciplinary VM. During the operation, the surgeon (H.T.) who had rehearsed the resection on Vitrea software before the actual surgery aimed to resect the expected volume determined from VM. During cardioplegic myocardial arrest, the resected myocardial volume was measured by liquid displacement, and additional resection was performed until the VM-predicted volume was resected. We have previously described our SM technique. ${ }^{13}$ In brief, under standard cardiopulmonary bypass (CPB) techniques with aortic/bicaval venous cannulation, transaortic SM was performed. More recently, use of 3D printing revealed a "septal band," a

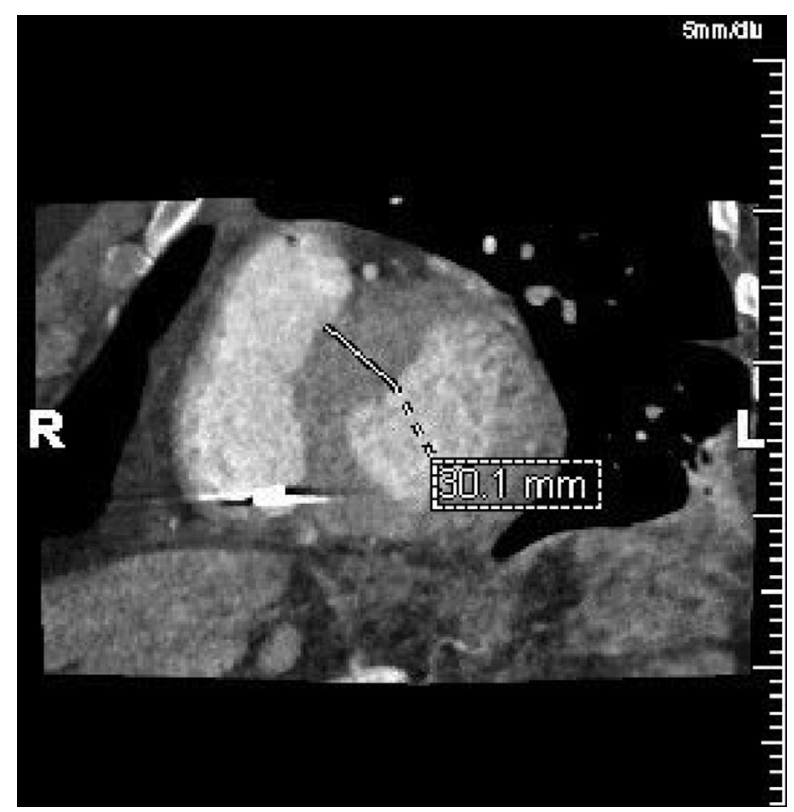

FIGURE 4. Short-axis view of left and right ventricle on gated computed tomography. The thickest point of left ventricular septum is measured. common anatomical feature of these patients. The thickened septum, visualized as a broad-based band in 3D-printed hearts, was found to start basally from underneath the left coronary annulus, then run anteriorly and obliquely in a clockwise fashion from base to apex down the septal wall, and reach down to the base of the posteromedial papillary muscles and mid inferior wall (Figure 6, Video 3). Our technique aims to resect this septal band in 3 steps: (1) resection of tissue via longitudinal parallel-guided incisions in the septum as described in the original Morrow procedure with extension toward the LV apex (Morrow part); (2) left-sided excision toward the left trigone (left lateral part); and (3) right-sided excision extending down to the base of the posterolateral papillary muscle (right lateral part). Bimanual examination was performed by palpating the septum before and after the resection with 2 fingers-the left index finger was inserted through the aortotomy for the left side of the septum and the right index finger through a small right atriotomy/tricuspid valve for the right septum. Mitral valve procedures such as abnormal chord resection, papillary muscle realignment, and leaflet plication were added as needed in selected cases. TEE-guided anatomical assessment and pressure measurement (with and without premature ventricular contraction) of the LVOT were performed before and after CPB to confirm the adequacy of myectomy.

\section{Statistical Analysis}

Statistical analysis was performed using Stata 14 software (Stata Corp, College Station, Tex). Continuous variables are reported as mean \pm standard deviation and compared with 2-sample $t$-tests. Categorical variables are summarized with percentages and analyzed using $\chi^{2}$ or Fisher exact test. Statistical significance is defined as a 2-sided $\alpha<0.05$. Pearson correlation was calculated between retrospective VM methods using actual myectomy as the gold standard.

\section{RESULTS}

\section{Baseline Characteristics}

Mean patient age was $52.1 \pm 10.6$ years. In total, $73 \%$ were female, and $80 \%$ were New York Heart Association class 3 or 4 . Preoperative TTE revealed a mean ejection fraction of $73.0 \pm 5.3 \%$, LV end diastolic dimension $3.9 \pm 0.4 \mathrm{~cm}$, LV septal thickness $2.1 \pm 0.5 \mathrm{~cm}$, LV posterior wall thickness $1.3 \pm 0.3 \mathrm{~cm}$, and peak LVOT gradient $79 \mathrm{~mm} \mathrm{Hg} \mathrm{(47} \mathrm{82)} \mathrm{at} \mathrm{rest} \mathrm{and} 90 \mathrm{~mm} \mathrm{Hg} \mathrm{(76-144)} \mathrm{with} \mathrm{Val-}$ salva maneuver. Five patients $(33 \%)$ had mitral regurgitation (MR) severity moderate $(2+)$ or greater.

\section{Outcomes of Surgical Myectomy}

SM was performed after gated CT and VM. Mean resected volume was $6.2 \pm 1.7 \mathrm{~cm}^{3}$. Mean CPB time and 

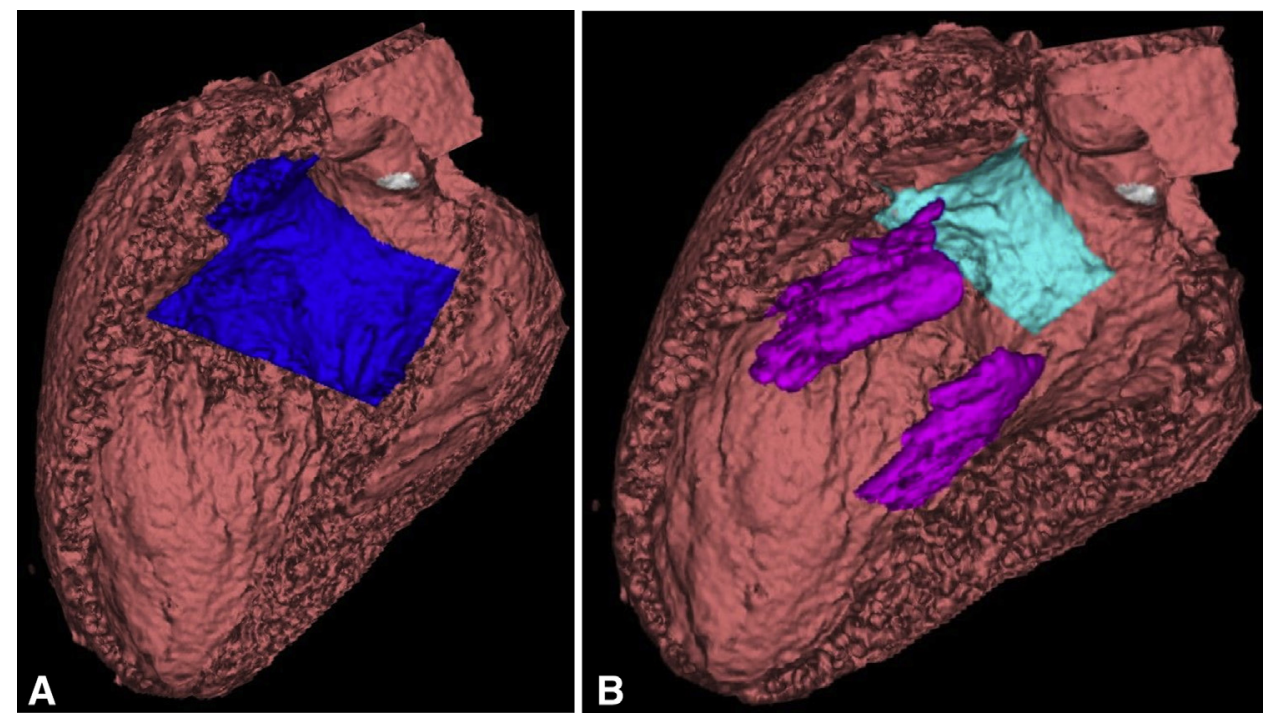

FIGURE 5. VM1 (ideal) and VM2 (conservative) resection visualized on lateral view of the left ventricle 3-dimensional reconstruction. A, VM1 (dark blue), which resected all asymmetric LV myocardium excluding $>1.5 \mathrm{~cm}$ distal to the head of the posterior papillary muscle and leftward of the left trigone anteriorly, avoiding the membranous septum and therefore His bundle proximally. B, VM2 (light blue) is limited apically and inferiorly by the proximal tip of the posteromedial papillary muscle head. In addition, the resection is no deeper than $1 \mathrm{~cm}$ in depth and leaves at least $1 \mathrm{~cm}$ of myocardial thickness. LV myocardium is shown in in soft pink; papillary muscles in bright pink; and membranous septum in white.

aortic crossclamp time were $122.5 \pm 20.7$ minutes and $86.0 \pm 12.6$ minutes, respectively. Concomitant mitral procedures were performed in 3 patients $(20 \%)$. Perioperative TEE was used. Preoperative and postoperative TEE revealed a significant reduction in LVOT gradient, which decreased from on average $80 \mathrm{~mm} \mathrm{Hg} \mathrm{(43-108)} \mathrm{to} 10 \mathrm{~mm}$

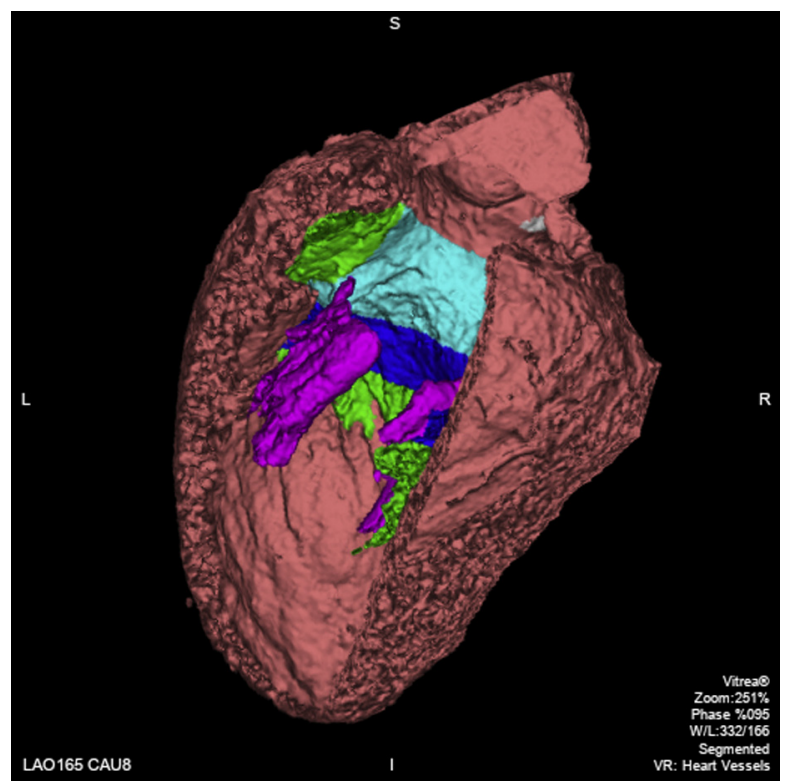

VIDEO 2. Total asymmetric left ventricular myocardium (green), ideal resection VM1 (dark blue), and conservative resection VM2 (light blue) overlaid. VM, Virtual myectomy. Video available at: https://www.jtcvs. org/article/S0022-5223(18)32934-9/fulltext.
$\mathrm{Hg}(8-12)(P<.001)$ and MR (frequency of patients with moderate + MR from $73 \%$ to $7 \%, P<.001)$.

\section{Postoperative Clinical Outcomes}

There were no in-hospital deaths. Patients underwent postoperative TTE after discharge for SM. Postoperative TTE revealed a mean ejection fraction of $65.4 \pm 4.1 \%$, LV end-diastolic diameter $4.2 \pm 0.5 \mathrm{~cm}$, septal thickness $1.4 \pm 0.2 \mathrm{~cm}$, posterior wall thickness $1.2 \pm 0.2 \mathrm{~cm}$, and peak LVOT gradient $13 \mathrm{~mm} \mathrm{Hg}$ (11-19) at rest and $20 \mathrm{~mm} \mathrm{Hg}(17-38)$ with Valsalva maneuver. No patient had moderate or greater MR.

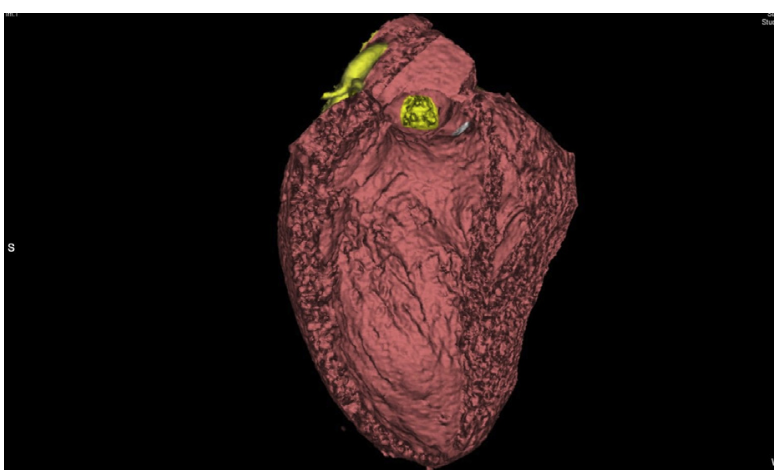

FIGURE 6. Thickened "septal band" visualized on lateral view of 3-dimensional reconstruction. Left ventricular myocardium is shown in soft pink. This is the hypertrophied myocardium that starts at the base anteriorly and extends in a clockwise fashion viewed from the base to apex in an apical fashion and is target for septal myectomy surgery. Membranous septum is shown in white and coronary arteries in yellow. 


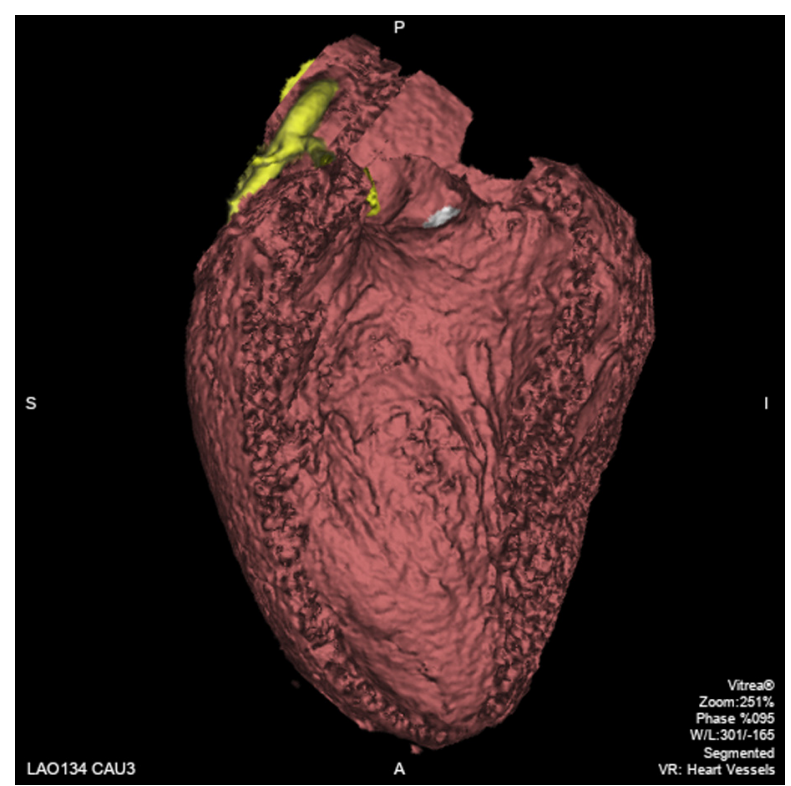

VIDEO 3. Basic anatomy of the septal band and its relationship with the rest of the left ventricular myocardium. Video available at: https://www. jtcvs.org/article/S0022-5223(18)32934-9/fulltext.

\section{Results of Cardiac CT and Retrospective VM}

Patients underwent a gated cardiac CT with mean phase of $94 \%$ (range $86 \%-98 \%$ ). Mean heart rate at time of CT was 60 beats per minute (range 53-75). Cardiac structures including coronary arteries, aortic valve cusps, left trigone, mitral leaflets, muscular septum, membranous septum, and the anterolateral and posteromedial papillary muscles were clearly visible in all cases. Mean total LV myocardial volume was $225.6 \mathrm{~cm}^{3}$ (range 161-411). Mean total asymmetric LV volume was $19.1 \mathrm{~cm}^{3}$ (range $5.2-48.7 \mathrm{~cm}^{3}$ ). Retrospective, blinded VM volumes with VM1 and VM2 were a mean of $12.9 \mathrm{~cm}^{3}$ (range $7.5-29.2 \mathrm{~cm}^{3}$ ) and $6.7 \mathrm{~cm}^{3}$ (range 3.5-13.2 $\mathrm{cm}^{3}$ ), respectively. Mean discrepancy with actual resection volume was $+6.7 \mathrm{~cm}^{3}$ (range -0.5 to $20.2 \mathrm{~cm}^{3}$ ) and $+0.5 \mathrm{~cm}^{3}$ (range -4.5 to $5.9 \mathrm{~cm}^{3}$ ) respectively (Figure 7), demonstrating increasingly conservative parameters from VM1 to VM2 showed improved mean discrepancy from actual surgical resection. The correlation between these 2 virtual myectomies and actual resected volume using Pearson correlation was weak $(\mathrm{R}=0.44)$ and moderate $(\mathrm{R}=0.56)$, respectively.

These measurements were compared with the septal thickness, which is the most common parameter to represent the degree of hypertrophy (Figure 8). Correlation between septal thickness and actual resection volume was very poor $\left(R^{2}=0.06\right)$, suggesting the need for better parameters to guide myectomy. There was moderate correlation between septal thickness measured on preoperative TTE to VM1 $\left(\mathrm{R}^{2}=0.47\right)$ and VM2 $\left(\mathrm{R}^{2}=0.60\right)$ resection.

\section{DISCUSSION}

Surgery for obstructive HCM remains an "art," with significant variation in technique and outcomes between institutions and operators. Despite this, surgery remains the backbone of treatment for medically refractory obstructive HCM. When performed in high-volume centers, surgical resection has excellent clinical outcomes with low morbidity and mortality. ${ }^{14}$ However, no objective method

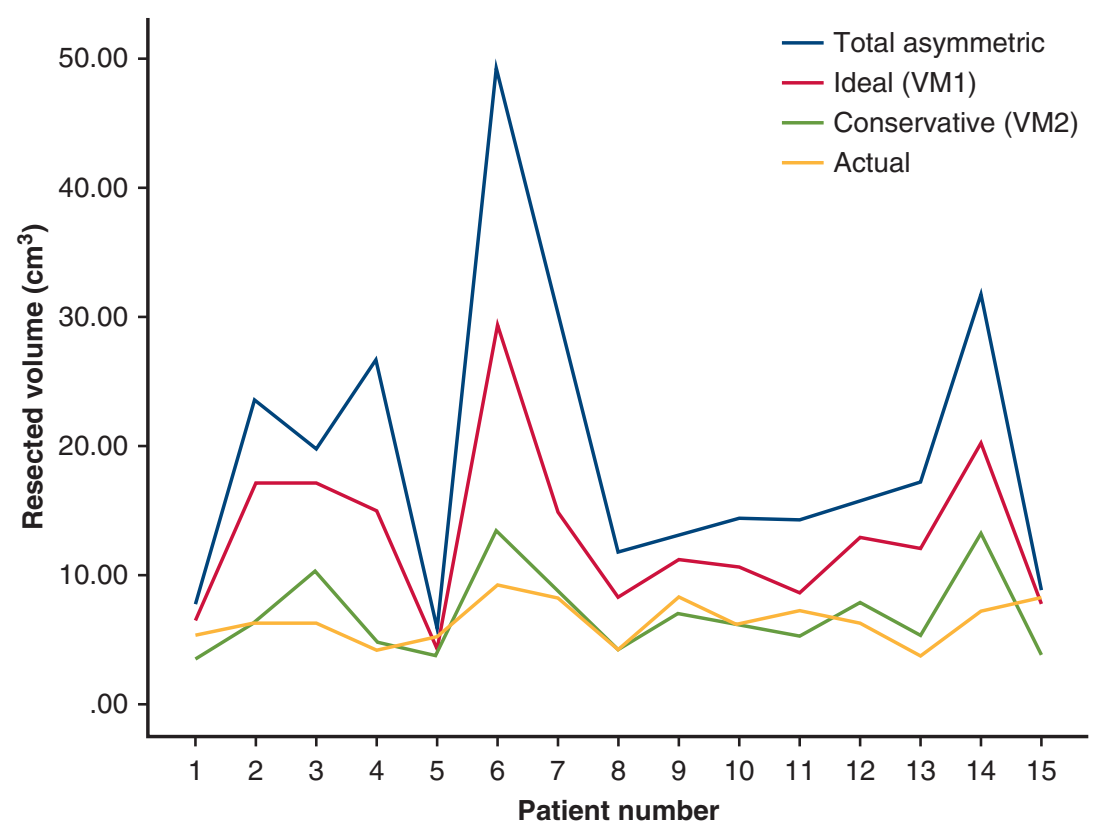

FIGURE 7. Total asymmetric volume (blue), idealized VM1 (red), conservative VM2 (green), and actual (yellow) resection volumes of each patient in study cohort is represented in this figure. $V M$, Virtual myectomy. 

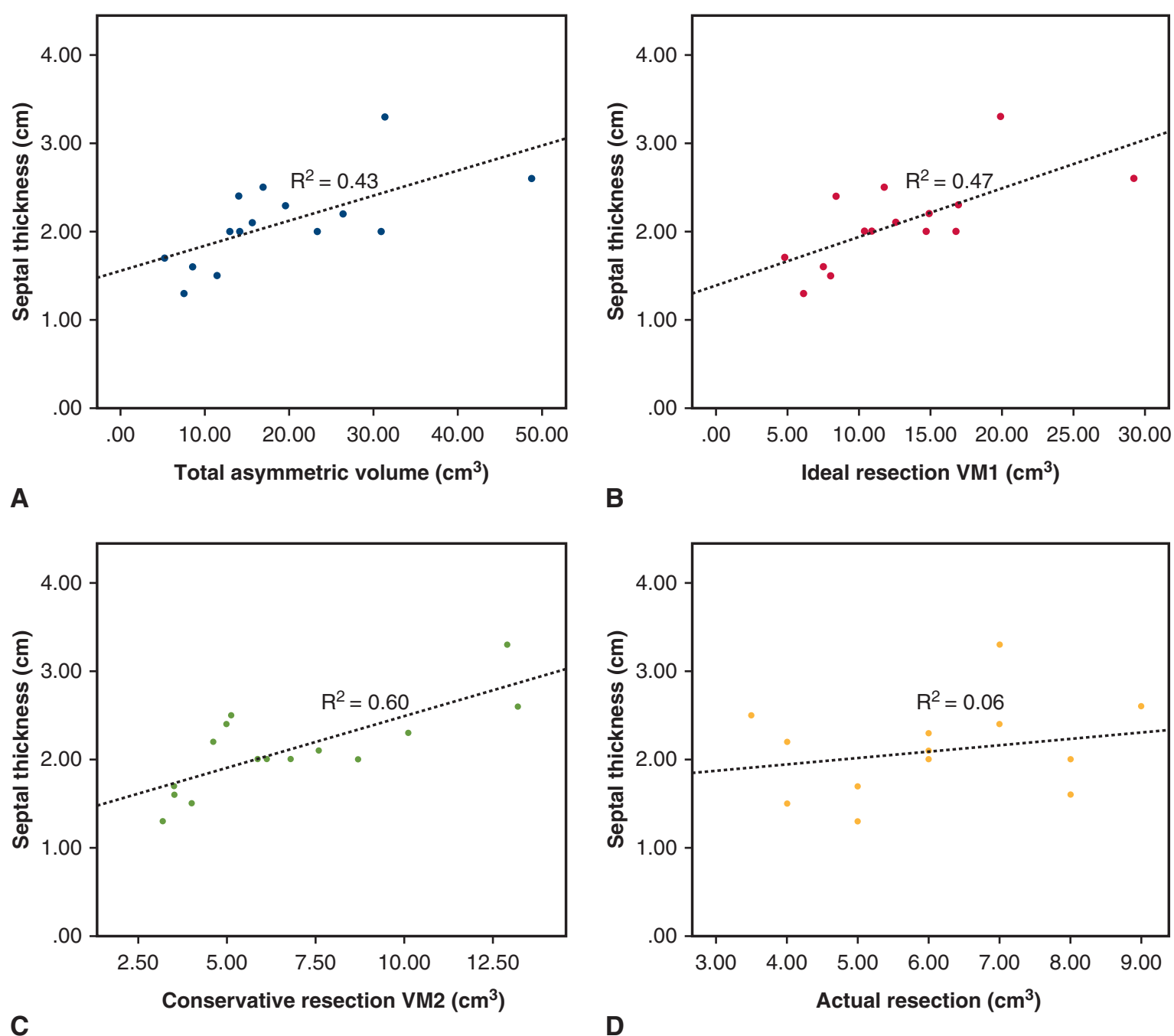

FIGURE 8. The correlation of A, total asymmetric volume; B, ideal resection (VM1); C, conservative resection (VM2); and D, actual resection with septal thickness on preoperative transthoracic echocardiogramis represented. VM, Virtual myectomy.

currently exists to predict the necessary extent of myectomy. Instead, much relies on surgical experience and expertise for intraoperative decision making. Visual inspection and manual examination of the septum are used to assess the resection extension during cardioplegic myocardial arrest ${ }^{15-17}$ with the aim to safely relieve obstruction by resecting sufficient hypertrophied myocardium while avoiding complications such as ventricular septal defect, heart block, or free wall rupture. Our study demonstrates that multidisciplinary assessment using VM before surgery can be a viable addition to these current tools (Video 4). Using gated cardiac CT with 3D reconstruction, we were able to reproduce the appearance of the heart under cardioplegia in diastolic arrest, $100 \%$ of relevant surgical landmarks in all patients were seen preoperatively. Postoperative discussions with the surgeon (H.T.) indicate that preoperative VM was helpful in determining how much myocardium to resect as well as clarifying surgical landmarks and anatomy during SM. In addition, other benefits of the 3D CT included demonstrating the detail of regional difference of septal thickness and width of the "septal band." Although these beneficial aspects were not examined specifically, we believe they contributed to achieving a satisfactory clinical outcome, and future studies should include these important aspects.

When retrospective, blinded VM was performed, we made several observations. Although the VM-predicted volume was removed during the actual myectomy (data not shown due to lack of prospective data collection), retrospective VM1 (idealized resection) and VM2 (conservative resection) overestimated actual resection by a mean of $6.7 \mathrm{~cm}^{3}$ and $0.5 \mathrm{~cm}^{3}$, respectively. There was a weak correlation with actual resection by VM1 and modest correlation by VM2. These findings suggest that VM1 is more 


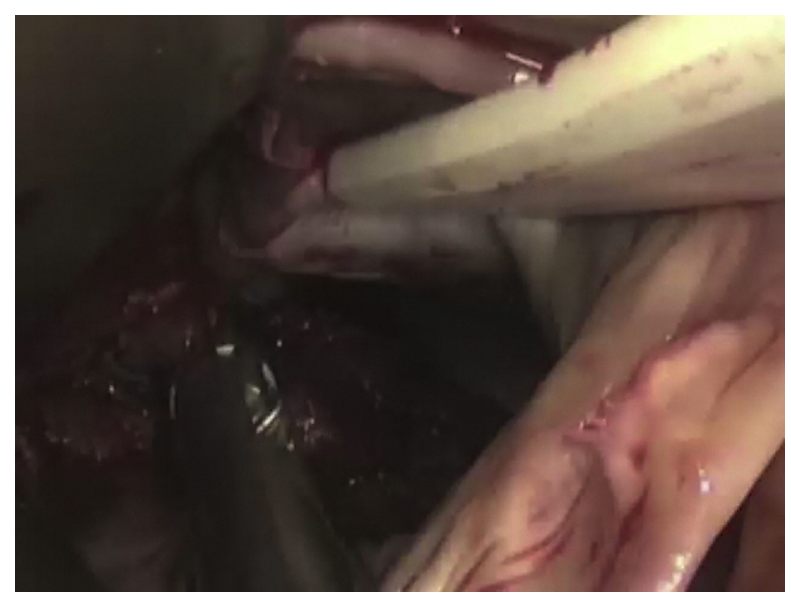

VIDEO 4. An explanation of our surgical and virtual myectomy technique. Video available at: https://www.jtcvs.org/article/S00225223(18)32934-9/fulltext.

"aggressive" than actual SM, whereas VM2 was much closer to the real resection. Other factors may have impacted the surgeon's ability or desire to resect what was considered an optimal amount (VM1), including the impact of preoperative multidisciplinary VM, visibility of hypertrophied LV through limited transaortic field, surgical concerns regarding safety, and depth of resection. VM2, whereas considered "conservative" objectively, had modest correlation and only overestimated the true resection by a small amount; keeping in mind the actual resection did achieve good clinical response. We also acknowledge despite VM1 being agreed as an "optimal" resection, this is speculative, and further study is required to determine the true anatomical extent of the "optimal" myectomy. Nevertheless, aiming for a greater resection volume may encourage adequate SM resection volumes and may lead to fewer cases with residual obstruction. Importantly, our surgical outcomes were measured only through short-term outcome analysis, and it is therefore possible that the actual resection volumes may not have been sufficient. We plan to continue long-term data collection on these patients.

In comparison with other preoperative planning techniques, gated cardiac CT with VM has some important advantages, although there are also additional risks, including contrast nephropathy and radiation exposure. Gated CT is widely available and has excellent spatial and temporal resolution. When performed using a standardized technique, all relevant intracardiac structures are seen. CT enables additional postprocessing using 3D reconstruction of any acquired cardiac phase. The reconstructed heart can be viewed from any direction using current-generation software. In addition, gated CT can be performed in arrhythmias such as atrial fibrillation and in the presence of intracardiac implantable devices.

In comparison, cardiac MRI lacks the degree of spatial resolution available in all 3 dimensions of cardiac CT; therefore, finer structures such as coronary arteries or the membranous septum may not be as easily visualized. Additional patient limitations such as claustrophobia and arrhythmias may be encountered with cardiac MRI. The presence of intracardiac devices may contraindicate MRI, or even if MRI compatible may generate significant artifact limiting ability to interpret studies. TTE has limitations related to available acoustic windows, tissue penetration, and cannot be reconstructed reliably in 3 dimensions. 3D printing to practice myectomy has been considered an alternative technique, ${ }^{18}$ although the time required to obtain a 3D-printed structure and expense of printing may be limiting factors.

There are a number of limitations to this study. First, this is a single-institution series with a small sample size. The present study focused on describing our initial clinical success, defined by our routine parameters including patient's status and echocardiographic results, without any control group. Also, the present study mostly focused on volume measurement; however, resecting appropriate amount of myocardium is only a part of the successful conduction of the procedure. For example, the current VM does not allow assessment of systolic anterior motion. We have initiated the next phase, that is, to obtain CT angiograms with whole cardiac cycle to further define the relevant anatomies, including the mitral valve as well as to obtain postoperative CT scans to better visualize the resection status of the intended area.

Second, as described previously, our preoperative VM remains somewhat subjective to the multidisciplinary team at our institution, and its technique was not standardized during this early learning phase, which may limit its widespread applicability. Third, the suitability of "optimal" VM (our retrospective VM1) versus VM2 (conservative resection) remains to be determined, and the impact of this resection at SM should be determined prospectively. Lastly, with the described protocol, the appearance of systolic anterior motion and area of septal/mitral contact was not assessed, although this could be addressed by including the complete cardiac cycle in the acquired CT imaging, at the cost of increased radiation exposure. Functional information such as LVOT gradient and severity of MR are not well assessed with CT, although typically this information is readily available with accompanying echocardiographic imaging. All of these limitations call for a prospective, multicenter study that includes a greater number of patients as well as HCM experts.

\section{CONCLUSIONS}

Gated cardiac CT with 3D reconstruction and VM is a novel imaging technique to demonstrate patient-specific anatomical details and assist in procedural planning. Clinical outcomes using preoperative VM to guide SM were excellent. This technique offers superior visualization of relevant static anatomy compared with other imaging 
modalities and shows potential to form a basis for objective preoperative assessment of HCM. However, further effort is required to better standardize and generalize the VM procedure. Nevertheless, the present study is the first to provide preliminary evidence that suggests feasibility and utility of 3D CT and VM in SM.

\section{Conflict of Interest Statement}

Authors have nothing to disclose with regard to commercial support.

\section{References}

1. Maron BJ, Peterson EE, Maron MS, Peterson JE. Prevalence of hypertrophic cardiomyopathy in an outpatient population referred for echocardiographic study. Am J Cardiol. 1994;73:577-80.

2. Minakata K, Dearani JA, Schaff HV, O'Leary PW, Ommen SR, Danielson GK. Mechanisms for recurrent left ventricular outflow tract obstruction after septal myectomy for obstructive hypertrophic cardiomyopathy. Ann Thorac Surg. 2005;80:851-6.

3. Ommen SR, Maron BJ, Olivotto I, Maron MS, Cecchi F, Betocchi S, et al. Longterm effects of surgical septal myectomy on survival in patients with obstructive hypertrophic cardiomyopathy. J Am Coll Cardiol. 2005;46:470-6.

4. Gersh BJ, Maron BJ, Bonow RO, Dearani JA, Fifer MA, Link MS, et al. 2011 ACCF/AHA guideline for the diagnosis and treatment of hypertrophic cardiomyopathy: executive summary. J Thorac Cardiovasc Surg. 2011;142:1303-38.

5. Marwick TH, Stewart WJ, Lever HM, Lytle BW, Rosenkranz ER, Duffy CI, et al. Benefits of intraoperative echocardiography in the surgical management of hypertrophic cardiomyopathy. J Am Coll Cardiol. 1992;20:1066-72.

6. Cho YH, Quintana E, Schaff HV, Nishimura RA, Dearani JA, Abel MD, et al. Residual and recurrent gradients after septal myectomy for hypertrophic cardiomyopathy-Mechanisms of obstruction and outcomes of reoperation. J Thorac Cardiovasc Surg. 2014;148:909-16.

7. Dearani JA, Ommen SR, Gersh BJ, Schaff HV, Danielson GK. Surgery insight: septal myectomy for obstructive hypertrophic cardiomyopathy-the Mayo Clinic experience. Nat Clin Pract Cardiovasc Med. 2007;4:503-12.
8. Swistel DG, Balaram SK. Surgical myectomy for hypertrophic cardiomyopathy in the 21st century, the evolution of the "RPR" repair: resection, plication, and release. Prog Cardiovasc Dis. 2012;54:498-502.

9. Ferrazzi P, Spirito P, Iacovoni A, Calabrese A, Migliorati K, Simon C, et al Transaortic chordal cutting: mitral valve repair for obstructive hypertrophic cardiomyopathy with mild septal hypertrophy. J Am Coll Cardiol. 2015;66: 1687-96.

10. Maron BJ, Maron MS. The remarkable 50 years of imaging in HCM and how it has changed diagnosis and management: from M-mode echocardiography to CMR. JACC Cardiovasc Imaging. 2016;9:858-72.

11. Rickers C, Wilke NM, Jerosch-Herold M, Casey SA, Panse P, Panse N, et al. Utility of cardiac magnetic resonance imaging in the diagnosis of hypertrophic cardiomyopathy. Circulation. 2005;112:855-61.

12. Maron MS. The current and emerging role of cardiovascular magnetic resonance imaging in hypertrophic cardiomyopathy. J Cardiovasc Transl Res. $2009 \mathrm{c} ; 2: 415-25$.

13. Fukuhara S, Edwards S, Kurlansky P, Takayama H. Bimanual examination for septal myectomy for hypertrophic cardiomyopathy. Interact Cardiovasc Thorac Surg. 2014;19:735-7.

14. Maron BJ, Dearani JA, Maron MS, Ommen SR, Rastegar H, Nishimura RA, et al. Why we need more septal myectomy surgeons: an emerging recognition. $J$ Thorac Cardiovasc Surg. 2017; 154:1681-5.

15. Dowling RD, Landreneau RJ, Gasior TA, Ziady GM, Armitage JM. Septal myectomy with a carbon dioxide laser for hypertrophic cardiomyopathy. Ann Thorac Surg. 2017;55:1558-60.

16. Nagueh SF, Buergler JM, Quinones MA, Spencer WH, Lawrie GM. Outcome of surgical myectomy after unsuccessful alcohol septal ablation for the treatment of patients with hypertrophic obstructive cardiomyopathy. J Am Coll Cardiol. 2007; 50:795-8.

17. Messmer BJ. Extended myectomy for hypertrophic obstructive cardiomyopathy Ann Thorac Surg. 1994;58:575-7.

18. Hermsen JL, Burke TM, Seslar SP, Owens DS, Ripley BA, Mokadam NA, et al Scan, plan, print, practice, perform: development and use of a patient-specific 3 dimensional printed model in adult cardiac surgery. J Thorac Cardiovasc Surg. 2017; 153:132-40.

Key Words: virtual myectomy, septal myectomy, hypertrophic cardiomyopathy 